\begin{tabular}{|c|l|}
\hline Title & $\begin{array}{l}\text { Unconventional Surface Impedance of a Normal-Metal Film Covering a Spin-T Triplet Superconductor Due to Odd- } \\
\text { Frequency Cooper Pairs }\end{array}$ \\
\hline Author(s) & A sano, Y asuhiro; Golubov, A lexander A.; Fominov, Y akov V .; Tanaka, Y ukio \\
\hline Citation & $\begin{array}{l}\text { Physical Review Letters, 107(8), 087001 } \\
\text { https://doi.org/10.1103/PhysRevLett.107.087001 }\end{array}$ \\
\hline Issue Date & 2011-08-19 \\
\hline Doc URL & http://hdl.handle.net/2115/47059 \\
\hline Rights & ○ 2011 A merican Physical Society \\
\hline Type & article \\
\hline File Information & PRL107-8_087001.pdf \\
\hline
\end{tabular}

Instructions for use 


\title{
Unconventional Surface Impedance of a Normal-Metal Film Covering a Spin-Triplet Superconductor Due to Odd-Frequency Cooper Pairs
}

\author{
Yasuhiro Asano, ${ }^{1}$ Alexander A. Golubov, ${ }^{2}$ Yakov V. Fominov, ${ }^{3}$ and Yukio Tanaka ${ }^{4}$ \\ ${ }^{1}$ Department of Applied Physics and Center for Topological Science \& Technology, Hokkaido University, Sapporo 060-8628, Japan \\ ${ }^{2}$ Faculty of Science and Technology and MESA+ Institute of Nanotechnology, University of Twente, \\ 7500 AE, Enschede, The Netherlands \\ ${ }^{3}$ L. D. Landau Institute for Theoretical Physics, RAS, 119334 Moscow, Russia \\ ${ }^{4}$ Department of Applied Physics, Nagoya University, Nagoya 464-8603, Japan
}

(Received 7 October 2010; published 16 August 2011)

\begin{abstract}
We discuss the dynamic response of odd-frequency Cooper pairs to an electromagnetic field. By using the quasiclassical Green function method, we calculate the impedance $(Z=R-i X)$ of a normal-metal thin film which covers a superconductor. In contrast with the standard relation (i.e., $R \ll X$ ), the impedance in spin-triplet proximity structures shows anomalous behavior (i.e., $R>X$ ) in the low frequency limit. This unusual relation is a result of the penetration of odd-frequency pairs into the normal metal and reflects the negative Cooper pair density.
\end{abstract}

DOI: 10.1103/PhysRevLett.107.087001

PACS numbers: 74.25.nn, 74.45.+c, 74.70.-b

Exotic superconductors with an odd gap function $\Delta\left(\boldsymbol{k}, \omega_{n}\right)$ in the Matsubara frequency $\omega_{n}$ are called oddfrequency (odd- $\omega$ ) superconductors. The mysterious behavior of odd- $\omega$ Cooper pairs is an intriguing issue in the physics of superconductivity and superfluidity [1,2]. An odd- $\omega$ superconductor might not exhibit the essential properties of superconductivity such as the gapped quasiparticle spectrum and the standard diamagnetic Meissner effect. Unfortunately, the odd- $\omega$ pairing state has yet to be experimentally detected in bulk materials. Odd-frequency Cooper pairs themselves, however, may exist in superconducting proximity structures. It was theoretically predicted in ferromagnet/superconductor junctions that spin-mixing due to spin-dependent potential should generate odd- $\omega$ spin-triplet $s$-wave pairs [3-7]. Manifestations of triplet pairs were recently observed experimentally as a longrange Josephson coupling across ferromagnets [8-11]. An alternative way of creating odd- $\omega$ pairs was suggested in proximity structures involving a normal metal attached to an odd-parity spin-triplet superconductor that belongs to the conventional even-frequency symmetry class. The parity mixing due to inhomogeneity produces the odd- $\omega$ pairs [12].

Although the experiments [8-11] catch a sign of odd- $\omega$ pairs and theories [13-17] have predicted unusual properties of spin-triplet superconducting junctions, we have never had clear scientific evidence of odd- $\omega$ pairs. This is because physical values focused in these studies include only indirect information of the frequency symmetry. The present Letter shows that the surface impedance is a particular physical value which directly reflects the frequency symmetry of a Cooper pair. We predict anomalous features of a surface impedance which should make it possible to detect odd- $\omega$ pairs experimentally.
Surface impedance $Z=R-i X$ reflects the dynamic response of Cooper pairs to an electromagnetic field $[18,19]$. The surface resistance, $R$, corresponds to resistance due to normal electrons. In conventional superconductors, $R$ drops exponentially with decreasing temperature below superconducting transition temperature $T_{c}$. The reactance, $X$, represents power loss of the electromagnetic field due to Cooper pairs. Typical temperature dependence of the surface impedance in a conventional superconductor is shown in Fig. 1(a). The positive amplitude of the Cooper pair density guarantees a robust relation $R \ll X$ at low temperatures. The validity of the relation $R<X$, however, is questionable for odd- $\omega$ Cooper pairs because the odd- $\omega$ symmetry and negative pair density are inseparable from each other according to the standard theory of superconductivity [20]. In the present Letter, we conclude that the unusual relation $R>X$ can be observed in the presence of odd- $\omega$ Cooper pairs at low frequency and at low temperature.

Let us consider a bilayer of a superconductor and a thin normal-metal film as shown in Fig. 1(b), where $W$ is the

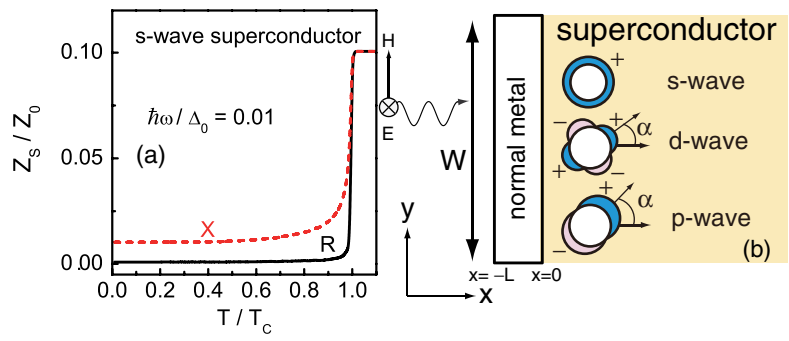

FIG. 1 (color online). (a) Typical behavior of the surface impedance in a conventional $s$-wave superconductor. (b) Proximity structure under consideration, where a thin film of a normal metal covers a superconductor. 
size of the superconductor and $L$ is the thickness of the normal metal. We mainly consider spin-triplet $p$-wave symmetry in the superconductor and compare results with those in spin-singlet $s$ - and $d$-wave symmetries. In all cases, the superconductor belongs to the conventional even-frequency symmetry class. The even- and odd-frequency pairs penetrate into the normal metal for the spin-singlet and spin-triplet cases, respectively $[13,14,21]$. We first solve the quasiclassical Usadel equation [22] in the standard $\theta$ parametrization [23], $\hbar D \nabla^{2} \theta(\boldsymbol{r}, \boldsymbol{\epsilon})+2 i \epsilon \sin \theta(\boldsymbol{r}, \boldsymbol{\epsilon})=0$, where $D$ is the diffusion constant of the normal metal and $\epsilon$ is the quasiparticle energy measured from the Fermi level. In what follows, we consider the normal-metal/superconductor (NS) junction in two dimensions as shown in Fig. 1(b). We choose $W \gg L$ so that we can neglect $y$ dependence of $\theta$. In the $p$-wave case, we assume that a spin-triplet Cooper pair consists of two electrons with opposite spin directionsthis assumption does not break the generality of the following argument. The Usadel equation is supplemented by the boundary condition at the NS interface which depends on the pairing symmetry of the superconductor [13,24,25], $\left.\partial_{x} \theta(x, \epsilon)\right|_{x=0}=\left(R_{D} / R_{B}\right)\left(\langle F\rangle / L T_{B}\right)$

$$
\langle F\rangle=\int_{-\pi / 2}^{\pi / 2} d \gamma \frac{T_{N} \cos \gamma\left(f_{s} \cos \theta_{0}-g_{s} \sin \theta_{0}\right)}{\left(2-T_{N}\right)+T_{N}\left(g_{s} \cos \theta_{0}+f_{s} \sin \theta_{0}\right)},
$$

where $\gamma$ is the incident angle of a quasiparticle measured from the $x$ axis, $\theta_{0}=\theta(x=0, \epsilon)$, and $R_{D}$ is the resistance of the normal metal. At the NS interface, a potential barrier described by $v_{0} \delta(x)$ suppresses the transmission probability $T_{B}=\int_{0}^{\pi / 2} d \gamma \cos \gamma T_{N}$ with $T_{N}=\cos ^{2} \gamma /\left(z_{0}^{2}+\cos ^{2} \gamma\right)$, $z_{0}=v_{0} /\left(\hbar v_{F}\right)$, and $v_{F}$ being the Fermi velocity. As a result, the resistance of the NS interface becomes $R_{B}=$ $\left[\left(e^{2} / \pi \hbar\right)\left(k_{F} W / \pi\right) T_{B}\right]^{-1}$. The Green function in the superconductor depends on $\gamma$ and the orientation angle $\alpha$ in Fig. 1(b) as $g_{ \pm}=\epsilon / \sqrt{\epsilon^{2}-\left|\Delta_{ \pm}\right|^{2}}$ and $f_{ \pm}=$ $i \Delta_{ \pm} / \sqrt{\epsilon^{2}-\left|\Delta_{ \pm}\right|^{2}}$, where $\Delta_{ \pm}=\Delta \Psi\left(\gamma_{ \pm}\right)$with $\Delta$ being the amplitude of the pair potential, $\gamma_{+}=\gamma-\alpha$ and $\gamma_{-}=$ $\pi-\gamma-\alpha$. The form factor $\Psi(\gamma)$ characterizes the pairing symmetry as $\Psi(\gamma)=1, \cos \gamma$, and $\cos 2 \gamma$ for the $s^{-}, p-$, and $d$-wave symmetries, respectively. At a particular orientation angle, the $p$ wave with $\alpha=0, p$ wave with $\alpha=\pi / 2, d$ wave with $\alpha=0$, and $d$-wave with $\alpha=\pi / 4$ correspond to the $p_{x}, p_{y}, d_{x^{2}-y^{2}}$, and $d_{x y}$ symmetry, respectively. In Eq. (1), $g_{s}=\left(g_{+}+g_{-}\right) / \xi$ with $\xi=1+$ $g_{+} g_{-}+f_{+} f_{-}$, and $f_{s}=\left(f_{+}+f_{-}\right) / \xi$ for the spin-singlet pairing symmetry, while $f_{s}=i\left(f_{+} g_{-}-f_{-} g_{+}\right) / \xi$ for the spin-triplet one $[13,25]$. For the chiral $p$-wave symmetry, when $\Psi(\gamma)=e^{i \gamma}, \theta$ obeys a boundary condition which is slightly different from Eq. (1) [14]. At the outer surface of the normal metal, we require $\left.\partial_{x} \theta(x, \epsilon)\right|_{x=-L}=0$. The retarded Green functions are obtained as $g(x, \epsilon)=$ $\cos \theta(x, \epsilon)$ and $f(x, \epsilon)=\sin \theta(x, \epsilon)$.
Having found the Green functions, we can calculate the local complex conductivity $\sigma_{N}(x, \omega)=\sigma_{1}+i \sigma_{2}$ which is represented only by the retarded Green function as [26]

$$
\begin{gathered}
\frac{\sigma_{1}(x, \omega)}{\sigma_{0}}=\frac{1}{2 \hbar \omega} \int_{-\infty}^{\infty} d \epsilon[J(\epsilon+\hbar \omega)-J(\epsilon)] K_{1}, \\
\frac{\sigma_{2}(x, \omega)}{\sigma_{0}}=\frac{1}{2 \hbar \omega} \int_{-\infty}^{\infty} d \epsilon\left[J(\epsilon+\hbar \omega) K_{2}+J(\epsilon) K_{3}\right], \\
K_{1}=f_{I}(\epsilon) f_{I}(\epsilon+\hbar \omega)+g_{R}(\epsilon) g_{R}(\epsilon+\hbar \omega), \\
K_{2}=f_{R}(\epsilon) f_{I}(\epsilon+\hbar \omega)-g_{I}(\epsilon) g_{R}(\epsilon+\hbar \omega), \\
K_{3}=f_{R}(\epsilon+\hbar \omega) f_{I}(\epsilon)-g_{I}(\epsilon+\hbar \omega) g_{R}(\epsilon),
\end{gathered}
$$

with $J(\epsilon)=\tanh \left(\epsilon / 2 k_{B} T\right), g_{R}(\epsilon)=\operatorname{Re}[g(x, \epsilon)], g_{I}(\epsilon)=$ $\operatorname{Im}[g(x, \boldsymbol{\epsilon})], f_{R}(\boldsymbol{\epsilon})=\operatorname{Re}[f(x, \boldsymbol{\epsilon})]$, and $f_{I}(\boldsymbol{\epsilon})=\operatorname{Im}[f(x, \boldsymbol{\epsilon})]$. The local impedance in the normal metal is calculated from the complex conductivity as

$$
Z_{N}(x, \omega)=R_{N}-i X_{N}=(1-i) \sqrt{\frac{\hbar \omega}{\Delta_{0}} \frac{\sigma_{0}}{\sigma_{N}(x, \omega)}} Z_{0},
$$

where $Z_{0} \equiv \sqrt{2 \pi \Delta_{0} / \sigma_{0} c^{2} \hbar}, \Delta_{0}$ is the amplitude of pair potential at $T=0$, and $\sigma_{0}$ is the Drude conductivity in the normal metal. The surface impedance in Fig. 1(a) is calculated from the above formula, taking the Green function of a uniform $s$-wave superconductor.

The theory basically includes three junction parameters: the thickness of the normal metal $L$, strength of the potential barrier $z_{0}$, and the diffusion constant in the normal metal $D$, which are converted to $R_{D} / R_{B}, E_{\mathrm{Th}}=\hbar D / L^{2}$, and $z_{0}$ with $E_{\mathrm{Th}}$ being the Thouless energy. As we discuss later on, the results shown below depend on these parameters only quantitatively. Thus we fix them at $R_{D} / R_{B}=1$, $E_{\mathrm{Th}}=0.5 \Delta_{0}$, and $z_{0}=2.5$ throughout this Letter, which leads to $T_{B} \approx 0.1$ and $L \approx 2.7 \xi_{T_{c}}$ with $\xi_{T_{c}}=$ $\sqrt{\hbar D / 2 \pi k_{B} T_{c}}$ being the coherence length. We add a small positive imaginary part $i \epsilon_{\lambda}$ to energy to provide the retarded causality and to obtain reasonable convergence of the integration in Eqs. (2) and (3). In real junctions, $i \epsilon_{\lambda}$ stems from inelastic scatterings of a quasiparticle. We fix $\epsilon_{\lambda}$ at $0.01 \Delta_{0}$.

First, we compare the retarded Green functions at the surface of the normal metal $(x=-L)$ for the $s$-wave symmetry in Figs. 2(a) and 2(b) with those for the chiral $p$ wave in (d) and (e). The real part of the normal Green function $g_{R}(\epsilon)$ is the quasiparticle density of states normalized by the normal density of states at the Fermi level. The normal Green function always satisfies $g(-\epsilon)=$ $[g(\epsilon)]^{*}$ irrespective of pairing symmetry as shown in (a) and $(\mathrm{d})$. In the $s$-wave case, $g_{R}(\epsilon)$ shows a minigap for $|\epsilon|<\Delta_{N} \sim 0.15 \Delta_{0}$. In the chiral $p$-wave case, $g_{R}$ in (d) shows a large peak around $\epsilon=0$, which can be understood 

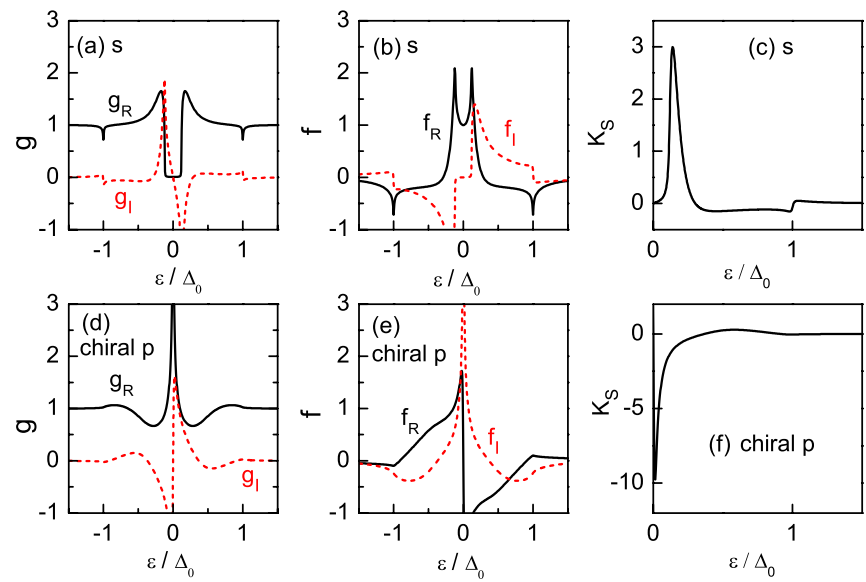

FIG. 2 (color online). The retarded Green functions at the surface of the normal metal for the $s$ wave (a),(b) and the chiral $p$-wave symmetry (d),(e). The solid and broken lines represents the real and imaginary part of the Green function, respectively. In (c) and (f), $K_{s}$ is the spectral pair density and corresponds to the kernel of the imaginary part of complex conductivity at $\omega \rightarrow 0^{+}$.

as the penetration of midgap Andreev resonant states [27] into the normal metal. The penetration of such states into a normal metal is possible only for spin-triplet junctions $[13,15]$. The feature of the anomalous function $f_{R}(\epsilon)$ depends on the pairing symmetry of the superconductor. The results in (b) show that $f_{R}(\epsilon)$ is an even function of $\epsilon$ and $f_{I}(\epsilon)$ is an odd function of $\epsilon$ (i.e., $\left.f(-\epsilon)=[f(\epsilon)]^{*}\right)$. This relation means the presence of the even-frequency Cooper pairs in the normal metal and always holds for spin-singlet NS structures. The results in (e), however, show $f(-\boldsymbol{\epsilon})=$ $\left.-[f(\epsilon)]^{*}\right)[21]$. This relation represents the penetration of odd-frequency Cooper pairs into the normal metal and holds for spin-triplet NS structures.

Second, we show the local impedance at the surface of the normal metal $Z_{N}(-L, \omega)$ as a function of temperature at a small $\hbar \omega$ fixed at $0.01 \Delta_{0}$ in Fig. 3. In the $s$-wave (a) and $d_{x^{2}-y^{2}}$ (b) symmetry, the results show conventional behavior totally consistent with those in Fig. 1(a). Namely, $R_{N}$ and $X_{N}$ satisfy the relation $R_{N} \ll X_{N}$. For $d_{x y}$ and $p_{y}$ symmetries, a relation $R=X$ always holds irrespective of $\omega$ and $T$ (results are not shown). This reflects the skin effect of the normal metal because there is no proximity effect in these symmetries $[25,28]$. In the chiral $p$-wave symmetry in (c), on the other hand, $R_{N}$ become larger than $X_{N}$ for $T<T_{c}$. A similar behavior can be seen also in the $p_{x}$-wave symmetry for $T<T^{*} \sim$ $0.4 T_{c}$ as shown in (d). The impedance clearly exhibits different characteristic behaviors depending on the pairing symmetry of the superconductor. In Figs. (c) and (d), $\sigma_{2}$ changes its sign to negative. It is easy to confirm that the sign change of $\sigma_{2}$ results in $R_{N}>X_{N}$ in Eq. (7). Thus $R_{N}>X_{N}$ is a robust and universal property of odd-frequency Cooper pairs.
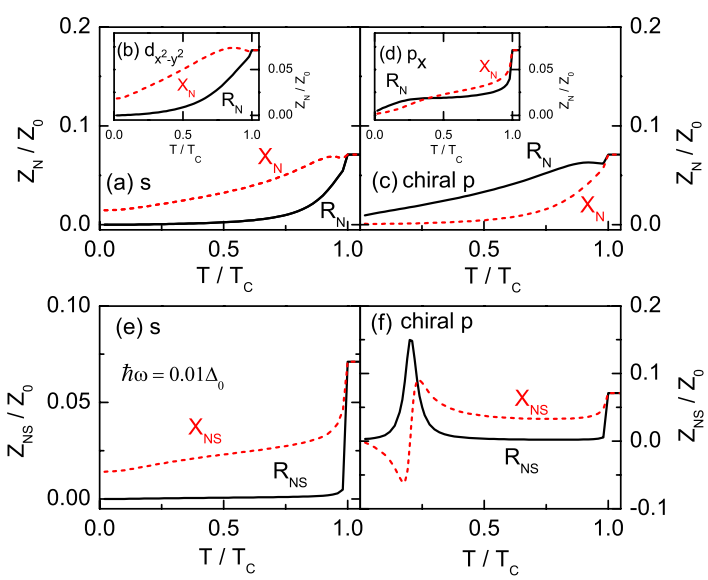

FIG. 3 (color online). Local impedance at a surface of the normal metal (i.e., $x=-L$ ) versus temperature at $\hbar \omega=$ $0.01 \Delta_{0}$ for (a) $s$-wave, (b) $d_{x^{2}-y^{2}}$-wave, (c) chiral $p$-wave, and (d) $p_{x}$-wave symmetry. The impedance of a NS bilayer versus temperature for (e) $s$-wave and (f) chiral $p$-wave symmetry.

To understand the characteristic feature of complex conductivity at small $\omega$, we analyze the spectral pair density defined by $K_{s}(\epsilon)=f_{R}(\epsilon) f_{I}(\epsilon)-g_{R}(\epsilon) g_{I}(\epsilon)=$ $\operatorname{Im} f^{2}(\epsilon)$ which appears in the integrand of $\sigma_{2}$ in Eq. (3) at very small $\omega$. The spectral pair density contains full information about the frequency symmetry of Cooper pairs. In the $s$-wave case, $K_{s}$ in Fig. 2(c) is mostly positive for $\epsilon>0$ and has a positive peak around $\epsilon=\Delta_{N}$. Since $K_{s}$ is an odd function of $\epsilon$, we only show the results for $\epsilon>0$. On the other hand in the chiral $p$-wave symmetry in Fig. 2(f), $K_{s}$ is mostly negative for $\epsilon>0$ and has a large negative peak near $\epsilon=0$ due to the odd-frequency symmetry. The Cooper pair density in the normal metal $n_{s}=$ $\int_{-\infty}^{\infty} d \epsilon J(\epsilon) K_{s}(\epsilon)$ and $\sigma_{2}$ are positive for the $s$-wave case. On the other hand, they are negative for the chiral $p$-wave case. Therefore we conclude that the negative $\sigma_{2}$ is the direct consequence of the odd-frequency symmetry of Cooper pairs. This argument generally holds when $\hbar \omega$ is smaller than all energy scales relating to the proximity effect. The smallest energy scale at the present calculation is the peak width of the Green function shown in Fig. 2. We have confirmed that $T^{*}$ in Fig. 3(d) increases to $T_{c}$ with decreasing $\omega$. The conclusion remains unchanged under varying the junction parameters $R_{D} / R_{B}, E_{\mathrm{Th}}$, and $z_{0}$, since they do not affect the frequency symmetry of Cooper pairs.

Next, we briefly discuss the physics behind the negative $\sigma_{2}$. The electric current in the normal metal may be described by two contributions: $\boldsymbol{j}=\boldsymbol{j}_{N}+\boldsymbol{j}_{S}$ with the normal current $\boldsymbol{j}_{N}=\sigma_{0} \boldsymbol{E}$ and the supercurrent $\boldsymbol{j}_{S}=$ $-\frac{n_{s} e^{2}}{m c} \boldsymbol{A}=i \frac{n_{s} e^{2}}{m \omega} \boldsymbol{E}$ with $m$ being the mass of electron [29]. The complex conductivity then becomes $\sigma_{1}+i \sigma_{2}=$ $\sigma_{0}+i \frac{n_{e} e^{2}}{m \omega}$. The positive value of $n_{s}$ is indispensable for explaining the Meissner effect. This argument can be easily confirmed by solving the magnetic field 
with $\boldsymbol{\nabla} \times \boldsymbol{H}=\frac{4 \pi}{c} \boldsymbol{j}_{S}$. In the case of odd-frequency pairs, however, $n_{s}$ becomes negative, which implies absence of the Meissner effect. This fact also significantly modifies the impedance of a NS bilayer defined by

$$
Z_{\mathrm{NS}}=R_{\mathrm{NS}}-i X_{\mathrm{NS}}=\bar{Z}_{N} \frac{Z_{S} \cos \bar{k}_{n} L-i \bar{Z}_{N} \sin \bar{k}_{n} L}{\bar{Z}_{N} \cos \bar{k}_{n} L-i Z_{S} \sin \bar{k}_{n} L},
$$

where $Z_{S}$ is the impedance of the superconductor shown in Fig. 1(a). Since $L$ being comparable to $\xi_{T_{c}}$, the conductivity depends on $x$ only slightly in the normal metal, which enables us to define spatially averaged values: the conductivity $\bar{\sigma}_{N}=\int_{-L}^{0} d x \sigma_{N}(x) / L$, the impedance $\bar{Z}_{N}=$ $-i \sqrt{4 \pi i \omega /\left(c^{2} \bar{\sigma}_{N}\right)}=\bar{R}_{N}-i \bar{X}_{N}$, and the wave number of the electromagnetic field $\bar{k}_{n}=\sqrt{i 4 \pi \omega \bar{\sigma}_{N} / c^{2}}$. The sign change of $\sigma_{2}$ results in the real value of $\bar{k}_{n}$ leading to the absence of the Meissner effect. In Fig. 3, we show the impedance of a NS bilayer for the $s$ wave in (e) and chiral $p$-wave NS junctions in (f), where we assume the resistivity of a normal metal $\rho=\sigma_{0}^{-1}$ is about $2 \times 10^{-6}[\Omega \cdot \mathrm{cm}]$. In the $s$-wave symmetry, the impedance of the NS bilayer [(e)] shows the conventional relation $R_{\mathrm{NS}}<X_{\mathrm{NS}}$. In the chiral $p$-wave symmetry, on the other hand, $R_{\mathrm{NS}}$ goes beyond $X_{\mathrm{NS}}$ at low temperature. In addition, the absence of the Meissner effect in the normal metal causes the sign change of $X_{\mathrm{NS}}$. Using relations $\bar{X}_{N} \ll \bar{R}_{N}$ and $Z_{S} \approx-i X_{S}$, it is possible to show $X_{\mathrm{NS}} \approx 0$ at $\bar{R}_{N} / X_{S}=\tan \left\{\operatorname{Re}\left(\bar{k}_{n} L\right)\right\}$.

Finally, we briefly discuss realistic structures for observing the unusual impedance. One possible example is a bilayer consisting of a normal metal and spin-triplet chiral $p$ wave superconductor $\mathrm{Sr}_{2} \mathrm{RuO}_{4}$ [30]. At the same time, the proposed effect can also be observed in superconductor/ferromagnet structures [3]. An example of this type is a bilayer of $\mathrm{Nb} / \mathrm{CrO}_{2}$, where only odd-frequency pairs can penetrate into the half-metallic ferromagnet $\mathrm{CrO}_{2}[4-6,8]$.

In summary, we have theoretically studied the impedance $(Z=R-i X)$ of a bilayer consisting of a normal metal and a superconductor. For spin-singlet superconducting proximity structures, we found the conventional and robust relation $R \ll X$. For spin-triplet ones, $R$ can be larger than $X$ and $X$ may change sign as a function of temperature at low $T$. On the basis of obtained results, we propose a method to analyze the spin symmetry of Cooper pairs in a superconductor and to detect odd-frequency superconductivity.

This work was supported by KAKENHI on Innovative Areas "Topological Quantum Phenomena" (No. 22103002). Ya. V.F. was supported by the RFBR (Grant No. 11-02-00077-a), the Russian Federal Agency of Education and Science (Contract No. P799), and the program "Quantum Physics of Condensed Matter" of the RAS.

[1] V.L. Berezinskii, JETP Lett. 20, 287 (1974).

[2] A. Balatsky and E. Abrahams, Phys. Rev. B 45, 13125 (1992).

[3] F. S. Bergeret, A. F. Volkov, and K. B. Efetov, Phys. Rev. Lett. 86, 4096 (2001); Rev. Mod. Phys. 77, 1321 (2005).

[4] Y. Asano, Y. Tanaka, and A. A. Golubov, Phys. Rev. Lett. 98, 107002 (2007).

[5] V. Braude and Yu. V. Nazarov, Phys. Rev. Lett. 98, 077003 (2007).

[6] M. Eschrig and T. Löfwander, Nature Phys. 4, 138 (2008).

[7] J. Linder et al., Phys. Rev. Lett. 102, 107008 (2009).

[8] R. S. Keizer et al., Nature (London) 439, 825 (2006).

[9] M. S. Anwar et al., Phys. Rev. B 82, 100501(R) (2010).

[10] T. S. Khaire et al., Phys. Rev. Lett. 104, 137002 (2010).

[11] J.W. A. Robinson, J.D. S. Witt, and M.G. Blamire, Science 329, 59 (2010).

[12] Y. Tanaka et al., Phys. Rev. Lett. 99, 037005 (2007); Y. Tanaka, Y. Tanuma, and A. A. Golubov, Phys. Rev. B 76, 054522 (2007).

[13] Y. Tanaka and S. Kashiwaya, Phys. Rev. B 70, 012507 (2004).

[14] Y. Tanaka et al., Phys. Rev. B 72, 140503(R) (2005).

[15] Y. Asano, Y. Tanaka, and S. Kashiwaya, Phys. Rev. Lett. 96, 097007 (2006).

[16] Y. Asano, Y. Tanaka, and A. A. Golubov, Phys. Rev. Lett. 98, 107002 (2007)

[17] Ya. V. Fominov, JETP Lett. 86, 732 (2007).

[18] D. C. Mattis and J. Bardeen, Phys. Rev. 111, 412 (1958).

[19] S. B. Nam, Phys. Rev. 156, 470 (1967).

[20] A. A. Abrikosov, L.P. Gorkov, and I. E. Dzyaloshinski, Methods of Quantum Field Theory in Statistical Physics (Dover, New York, 1975).

[21] Y. Tanaka and A. A. Golubov, Phys. Rev. Lett. 98, 037003 (2007).

[22] K. D. Usadel, Phys. Rev. Lett. 25, 507 (1970).

[23] In a normal metal, the anomalous Green function $\sin \theta$ corresponds to the $\hat{\tau}_{1}\left(\hat{\tau}_{2}\right)$ component of Nambu space in spin-triplet (spin-singlet) NS junctions.

[24] Yu. V. Nazarov, Phys. Rev. Lett. 73, 1420 (1994); Superlattices Microstruct. 25, 1221 (1999).

[25] Y. Tanaka, Yu. V. Nazarov, and S. Kashiwaya, Phys. Rev. Lett. 90, 167003 (2003)

[26] Ya. V. Fominov, M. Houzet, and L. I. Glazman (to be published).

[27] Y. Tanaka and S. Kashiwaya, Phys. Rev. Lett. 74, 3451 (1995).

[28] Y. Asano, Phys. Rev. B 64, 014511 (2001).

[29] M. Tinkham, Introduction to Superconductivity (Dover, New York, 2004).

[30] Y. Maeno et al., Nature (London) 372, 532 (1994). 